\title{
De la page au partage, du livre au live
}

Les blocs-notes de slameurs ou la petite fabrique d'oralittérature

\section{Camille Vorger}

\section{(2) OpenEdition}

Journals

Édition électronique

URL : http://journals.openedition.org/genesis/1118

DOI : 10.4000/genesis. 1118

ISSN : 2268-1590

Éditeur :

Presses universitaires de Paris Sorbonne (PUPS), Société internationale de génétique artistique littéraire et scientifique (SIGALES)

\section{Édition imprimée}

Date de publication : 15 novembre 2012

Pagination : 235-246

ISBN : 978-2-84050-869-4

ISSN : 1167-5101

\section{Référence électronique}

Camille Vorger, « De la page au partage, du livre au live », Genesis [En ligne], 35 | 2012, mis en ligne le 15 novembre 2014, consulté le 23 juin 2020. URL : http://journals.openedition.org/genesis/1118 ; DOI : https://doi.org/10.4000/genesis. 1118

Ce document a été généré automatiquement le 23 juin 2020.

Tous droits réservés 


\section{De la page au partage, du livre au live}

Les blocs-notes de slameurs ou la petite fabrique d'oralittérature

\section{Camille Vorger}

Je suis le meilleur ami des mots

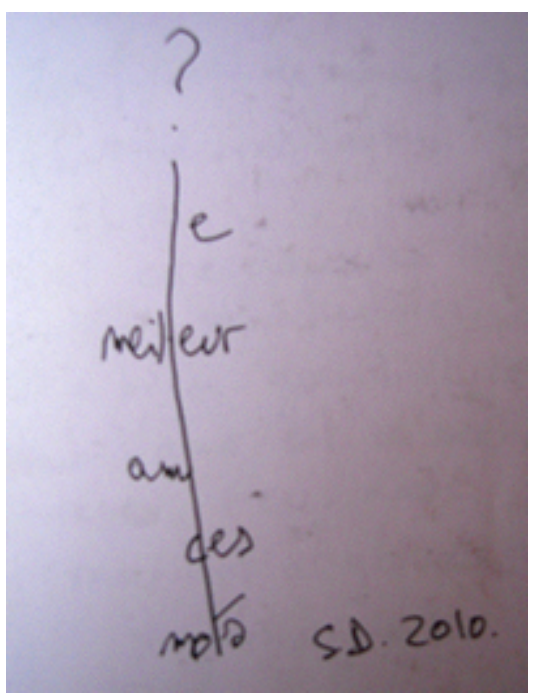

Papier à lettre hybride mi-homme mi-encre ${ }^{1}$

1 Se présentant comme néonomades ${ }^{2}$, les slameurs s'inscrivent dans la lignée des lointains aèdes, rhapsodes et autres troubadours : ils écrivent pour dire, visent à être entendus et l'œuvre n'advient pleinement que dans le contexte d'une performance (Zumthor, 1983). D'après la citation que nous avons choisi de mettre en exergue, le slameur se dit non seulement «meilleur ami des mots » mais aussi « mi-homme mi-encre », signifiant par là que sa performance, pour être vivante et mouvante, n'en est pas moins encrée ${ }^{3}$. Aussi, et si l'on se réfère à la définition par Almuth Grésillon de la critique génétique, le slam tel que nous l'allons explorer dans cet article en est l'objet idéal :

[la] critique génétique instaure un nouveau regard sur la littérature. Son objet : les

manuscrits littéraires, en tant qu'ils portent la trace d'une dynamique, celle du 
texte en devenir. Sa méthode : la mise à nu du corps et du cours de l'écriture, assortie de la construction d'une série d'hypothèses sur les opérations scripturales. Sa visée : la littérature comme un faire, comme activité, comme mouvement (1994, p. 7).

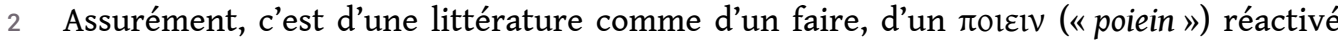
dans son sens original et sa connotation artisanale (d'où le terme d'atelier, que l'on retrouve à travers la formule semi-figée d'ateliers d'écriture) qu'il s'agit. D'un texte en devenir scénique, à l'instar du théâtre qui a déjà fait l'objet d'analyses génétiques", appelé à être incarné et porté par la voix du poète lors d'une déclamation publique. D'une dynamique langagière qui tend vers la créativité, l'innovation lexicale, comme l'ont montré nos précédentes études (2011). D'une écriture condensée, qui court sur le papier avant d'être dansée sur scène, d'une danse avec les mots et d'un flot intarissable qui se traduit précisément par la notion de flow, emprunté au technolecte du rap (Abry et Vorger, 2011).

3 Notre voyage au pays du slam nous a conduite à rencontrer de nombreux poètesslameurs et, à l'occasion d'entretiens, à découvrir certains de leurs écrits préparatoires, nous initiant par là même à cette «chasse au trésor » que représente l'aventure génétique :

La génétique des textes nous fait pénétrer dans le laboratoire secret de l'écrivain, dans l'espace intime d'une écriture qui se cherche. [...] c'est une recherche sur des indices matériels, une véritable enquête au cœur de l'écriture (de Biasi, 2000, p. 8, nous soulignons).

Parmi ces prémices d'un écrire pour dire définitoire du slam, nous pouvons distinguer ceux qui relèvent d'une phase prérédactionnelle (nous les appellerons « brouillons ", à la suite d'Almuth Grésillon, 1994), ceux qui s'inscrivent dans une phase proprement rédactionnelle - la rédaction pouvant être, en l'occurrence, partagée voire collective et ceux, plus rares, qui rendraient compte d'une ultime " mise au point » : ces derniers, répondant à l'appellation de «manuscrits » stricto sensu (voir infra, Nevchehirlian : « La mer »), ne sont guère représentatifs du slam qui désigne une forme de poésie orale en acte, et par conséquent, en mouvance perpétuelle. De fait, la forme actualisée que revêt un texte de slam lors d'une performance n'est jamais « ni tout à fait (la) même, ni tout à fait une autre " (Verlaine), même si la plupart des textes sont relativement stables et ont une trace écrite. Appréhendé dans tous ses états ${ }^{5}$, le slam a quelque chose de wharolien, tel un éternel avant-texte : "l'un est arrêté, l'autre en marche ", écrit Jean Bellemin-Noël, opposant en ces termes le Texte et l'avant-texte (1979, p. 116) ${ }^{6}$.

5 Après avoir apporté quelque éclairage et points d'ancrage de cette forme d'expression moderne - d'autant plus insaisissable qu'elle est fondamentalement plurielle - appelée «oralittérature» par le slameur qui se trouve au cœur du présent article, nous envisagerons ce que nous qualifions d'écriture palimpseste, à travers l'exemple illustré et commenté des blocs-notes de Souleymane Diamanka ${ }^{7}$. Enfin, nous aborderons les traces webuscrites de ce poète qui rendent compte d'un phénomène d'écriture partagée potentiellement emblématique du slam et de sa genèse. Au fil de cet article, nous croiserons des documents relevant de la genèse interne et des éléments de genèse externe approchés en entretien. 


\section{Le slam ou l'art de l'oralité manuscrite : éléments de définition et méthodologie}

6 À l'appellation d'« orature » proposée par Claude Hagège (1987, p. 110), à l'« oraliture » de Confiant et Chamoiseau (1999), nous préférons le concept d'oralittérature énoncé par le poète Souleymane Diamanka. Lors de l'entretien que ce dernier nous a accordé, il nous l'a explicité comme un entre-deux : «j'ai vraiment grandi avec les deux cultures aussi présentes l'une que l'autre: une éducation en peul et l'autre éducation en français. L'oralittérature, c'est l'oralité des contes, de mes parents, des proverbes, et tout ça. La littérature, c'est un peu ce que j'ai croisé à l'école ${ }^{8}$ ». Dans L'Hiver peul, il évoque "l'oralité manuscrite » chère à ses ancêtres, inhérente à la culture de ses origines et à l'art griotique :

Haal Pulaar peuple migrateur d'orateurs

Dont Amadou Hampaté Bâ a été l'ambassadeur

On passe à l'heure de l'oralité manuscrite... (2007)

7 Ces deux formules, que le slameur Grand Corps Malade résumera en un titre phare ("J'écris à l'oral», 2008), nous apparaissent emblématiques d'un slam où règne l'« écrire pour dire » et dont le principal trait définitoire est celui d'une poésie orale et publique, répondant à l'ambition première de son fondateur américain ${ }^{9}$ : celle de démocratiser et de moderniser la parole poétique. Pour autant, l'écriture n'est ni sacrifiée ni précipitée dans le slam français, même si une certaine condensation est généralement de mis $\mathrm{e}^{10}$ : longuement travaillée, lentement mûrie, et parfois mise en abyme dans les textes comme dans le slam de Grand Corps Malade cité ou encore dans cette autre formule du slameur de Saint-Denis : «La nuit, tous les stylos sont pris » (2008). Les entretiens que nous avons menés avec une vingtaine de slameurs français, suisse, québecois, espagnol et allemand, révèlent que pour la plupart d'entre eux, l'écriture demeure manuscrite, du moins lors de la phase prérédactionnelle. Le rapport au stylo, au bloc-notes, au carnet ou au cahier, au papier dans sa matérialité et son espace délimité par la page, s'avère fondamental et rares sont ceux qui s'adonnent d'emblée à une écriture tapuscrite :

C'est l'automne des blocs-notes. Beaucoup de feuilles sont mortes

J'ai trop de mots

Poésie trompe-l'œil... (Souleymane Diamanka, 2007, nous soulignons)

8 Le choix du terme de «blocs-notes »- retenu comme formule titulaire - par le poète ne rend pas compte de la réalité matérielle de l'objet réel (s'agissant plutôt de carnets) mais bien d'une dimension idéelle : à la différence du cahier ou du carnet, les feuilles du bloc-notes sont « volantes » et peuvent être détachées du support. D’où cet autre titre désignant le poème à travers la métaphore d'un «Papillon en papier» (voir infra). Poésie orale et aurale - volant de bouche à oreille -, portée par la voix, mais aussi poésie pour l'œil. En effet, la mise en espace du poème sur la page, et en amont, la mise en mots qui s'élabore progressivement et prend forme sur le blanc du papier, sur la trame d'un cahier d'écolier dont il tend précisément à s'échapper ${ }^{11}$, constitue un point d'appui, une aide à la représentation mentale voire à la mémorisation d'un texte destiné à être performé et parfois écrit dans cette seule perspective : texte qui doit, au dire des slameurs, "fonctionner » sur scène et dans le rapport à l'auditure (Bobillot, 2012). On assiste ainsi, naturellement, à une forme de projection de la voix dans certains manuscrits, dont témoigne le Marseillais Frédéric Nevchehirlian : «Regarde, il 
y a une construction, tout ça, c'est des dessins. Tu vois l'écriture explosée qui correspond à quelque chose. Parce que je peux écrire correctement, hein!», nous assure-t-il ${ }^{12}$. «Mots en liberté » chers aux Futuristes comme aux slameurs ${ }^{13}$.

Ce dernier manuscrit relève d'une forme quasi aboutie - et d'ailleurs " publiée » au sens premier de ce terme -, de la mise au point du poème, à la différence des avant-textes de Souleymane Diamanka analysés ci-après. De ce dernier, nous disposons de six extraits et échantillons préparatoires issus de blocs-notes, d'une double page qui rend compte d'un texte inédit, ainsi que d'une page de manuscrit résultant d'une écriture à deux mains (avec John Banzaii) : à la différence des autres documents cités et analysés dans cet article relevant d'une forme d'épitexte (Genette) - ils nous ont été révélés à l'occasion de l'entretien -, ce manuscrit est intégré au péritexte d'un recueil au titre éloquent : J'écris en français dans une langue étrangère (2007). Dans cet article, il nous faut donc " prendre en compte une poétique de l'écriture tout autant qu'une poétique du texte» (Debray-Genette, 1988), envisager le processus provisionnel en amont de la textualisation, tout en considérant les différents statuts conférés à ces écrits : qu'ils soient le fruit d'une écriture "en gestation ", la trace d'un poème nouveau-né et prêt à être oralisé, "baptisé sur scène ${ }^{14}$ », ou le support d'une écriture dialoguée/dialogique qui tend à intégrer l'autre ${ }^{15}$.

\section{Des mots tissés et métissés : des brouillons aux manuscrits}

\section{«Grifouillis » ou les prémices d'une écriture palimpseste}

Ces blocs-notes sont autant de traces de la genèse interne de l'œuvre de Souleymane Diamanka, œuvre-bijou en devenir puisque seule une partie infime en a été publiée à ce jour ${ }^{16}$. Ces " griffouillis » - selon le mot d'Aragon ${ }^{17}$ ou de Prévert ${ }^{18}$ - témoignent de jeux d'écriture auxquels le slameur se livre avec sérieux et assiduité, comme un musicien fait ses gammes, un chanteur ses vocalises et un danseur ses exercices d'assouplissement (d'après l'entretien cité). Ils révèlent une conception artisanale de l'écriture que partagent la plupart des slameurs. Slalomant entre des mots qu'elle tisse savamment, l'écriture s'élabore peu à peu, traits portraits ${ }^{19}$, pas à page, évoluant d'un "état sauvage " (Grésillon) à l'état scénique. La géographie du poème s'établit ainsi progressivement, les mots gagnant du terrain sur le blanc de la page où le dessein du poète prend forme :

C'est comme une peinture en fait, c'est-à-dire une première couche où j'écris vachement espacé, en me disant que je peux rajouter mes mots entre, au fur et à mesure, le temps passe et je vois où je rajoute des trucs. À la fin, visuellement ça dessine quelque chose (entretien cité).

11 Le slameur a été danseur et reste amateur de cultures urbaines. À la manière d'un graffeur, il aime à jouer avec le pouvoir magique de son pseudonyme (Billiez), de sa signature "Souley» qu'il rapproche du paronyme "soleil ${ }^{20}$ ", comme l'atteste la figure 1.

Fig. 1 


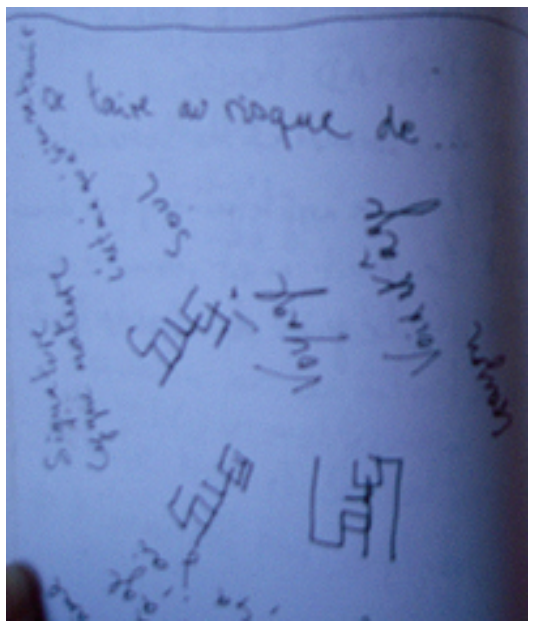

FIg. 1 : JEUX gRAPHIQUES AUTOUR DE LA SIgNATURE

12 Les lettres enchevêtrées traduisent la recherche d'effets visuels d'équivoque (Solex ?) qui sont le pendant de la paronomase (Souley/soleil). Appréhendés dans leur matérialité, qu'elle soit sonore ou graphique, les mots font l'objet de jeux perpétuels dont ces "mots croisés» (fig. 2), qui reflètent incidemment l'une des acceptions hors-champ du mot «slam ${ }^{21} »$. Au dire de l'un des activistes et pionniers du slam français, celui-là se définit comme Musique des lettres (Rouda). L'écriture se trouve ici délinéarisée et tend à devenir tabulaire :

L'espace manuscrit est délié de toute contrainte, l'écriture y évolue en toute liberté, la ligne horizontale y perd bien souvent ses droits, tant la vectorisation des graphismes peut être multiple (Grésillon, 1994, p. 51).

Fig. 2

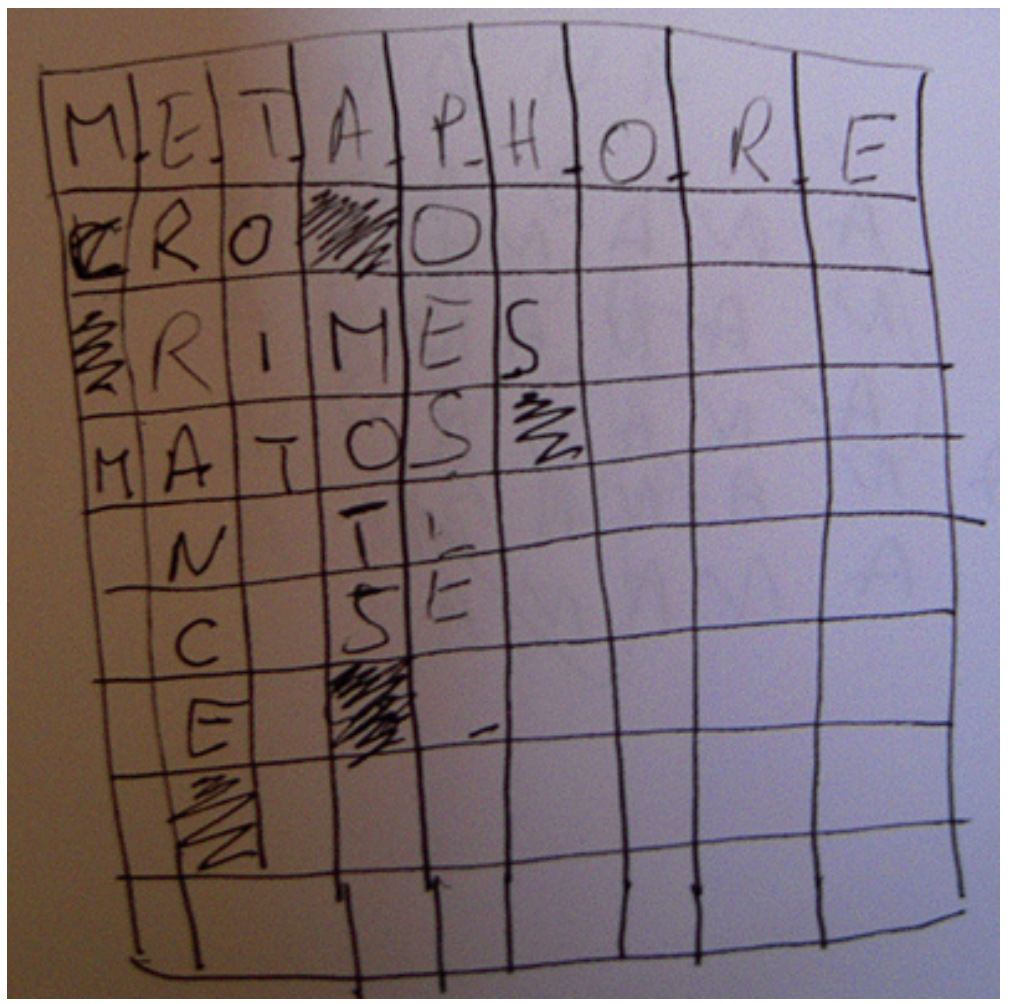

FIg. 2 : MOTS CROISÉS 
13 Comme l'illustre la figure 3, la recherche d'anagrammes est une constante chez cet artiste qui se dit d'ailleurs "métaphoriste", ce qui ne manque pas d'attirer notre attention sur l'importance de la métaphore ainsi que des aphorismes qu'il aime à détourner. De ce travail sur la texture sonore et sur l'architecture du texte naît cet autre néologisme : " architextural » (voir infra, fig. 5) traduit une écriture réflexive et créative en amont du texte, s'agissant en l'occurrence d'un mot composite obtenu par insertion de « texture » au formant directeur « architectural », en écho à l'architexte de Genette.

Fig. 3

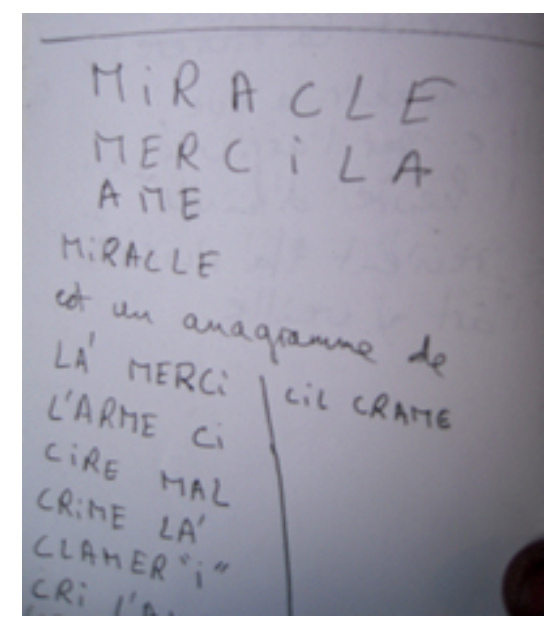

Fig. 3 : ANAgRAMMES

14 Outre les rayures (fig. 2) visant le noircissement de certaines cases à la manière des mots croisés, l'importance accordée à la rature (fig. 4) est une autre caractéristique majeure des brouillons ${ }^{22}$ de Souleymane Diamanka: "Je raye les mots pour qu'on les voie davantage ", disait Jean-Michel Basquiat auquel le poète aime à se référer ${ }^{23}$. De même, le slameur nous a avoué son souci de " raturer lisible " à l'aide d'un simple trait de biffure permettant de conserver les différentes strates de son texte, tel un graffiti qui se superpose à d'anciennes traces ${ }^{24}$. «Je respecte beaucoup les mots qu'il y a sous les ratures [...]. Les mots sous la rature sont super importants et souvent, quand on revient dessus, c'est qu'il y avait une puissance qui nous gênait peut-être, et qu'il faut laisser » (entretien cité). Pleinement conscient du potentiel de la rature comme « trésor

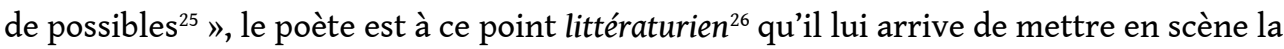
rature au cœur de son écriture (fig. 4). 
Fig. 4

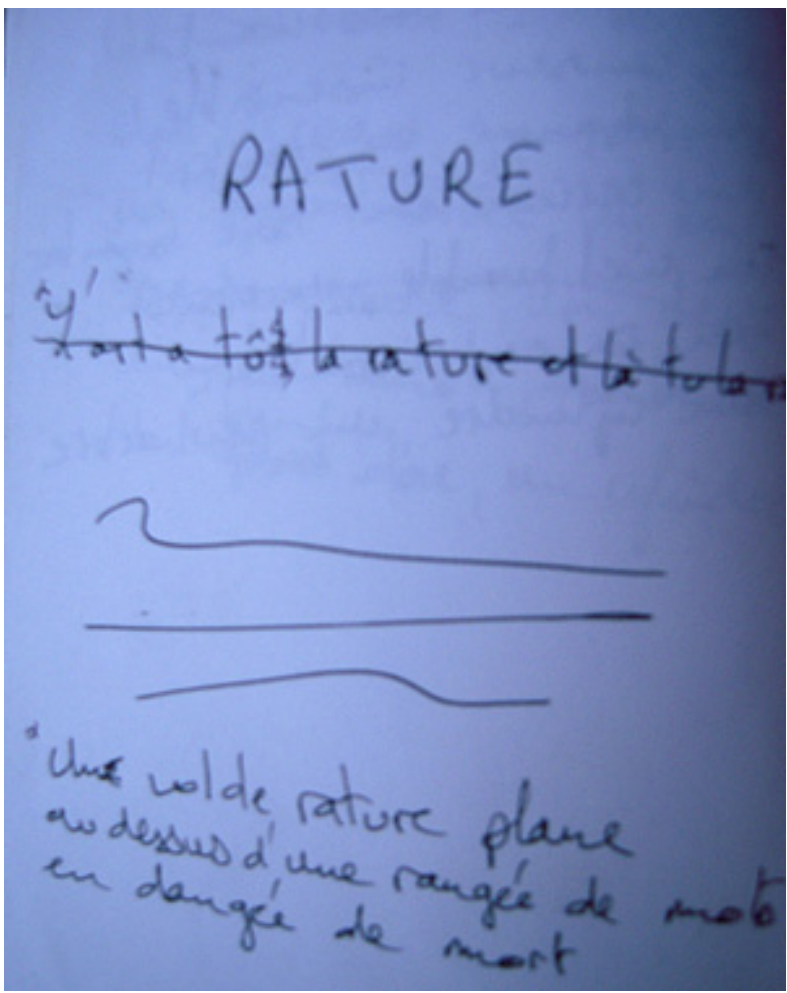

FIg. 4 : RATURE

15 Sa recherche d'holorimes et de palindromes (fig. 5 et 6) révèle une écriture mimétique : la rose des vents (suggérée lexicalement par la locution "aux quatre vents») matérialise ici le palindrome "rêver». Il s'agit d'un motif récurrent qui court sur plusieurs pages du bloc-notes. Une fois encore, le texte s'avère autotélique, se référant aux mots ("Ose ce mot»), ou encore à l'art urbain, relié à la culture hip-hop dont le poète est issu en tant qu'ex-rappeur. Il se reconnaît volontiers dans la formule d'« art ignare » emprunté à J.-M. Basquiat et reprise comme titre d'un texte sur son album : «J'ai toujours fait ce rêve et je remercie la rue. Tout ce que j'ai pu écrire, c'est sur ses lèvres que je l'ai lu... » (2007). 
Fig. 5

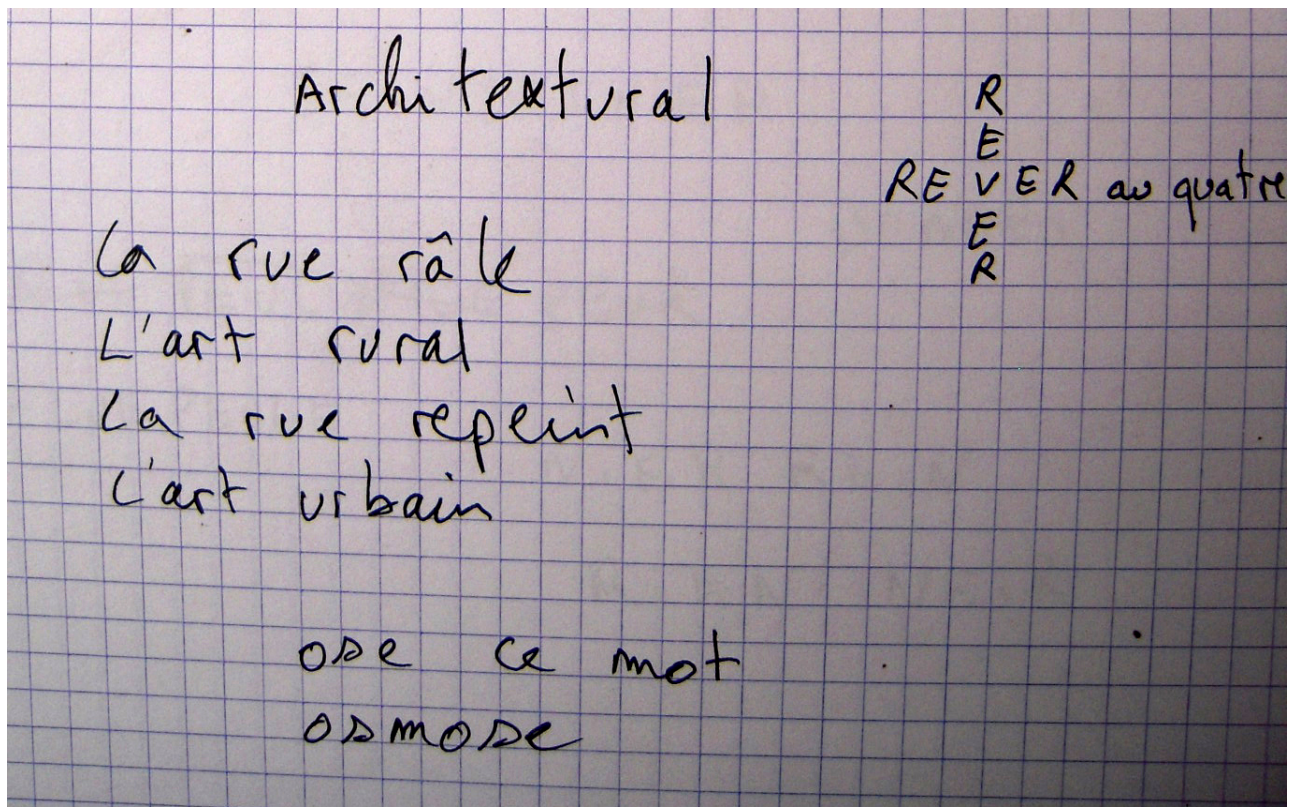

Fig. 5 : Architextural

Fig. 6

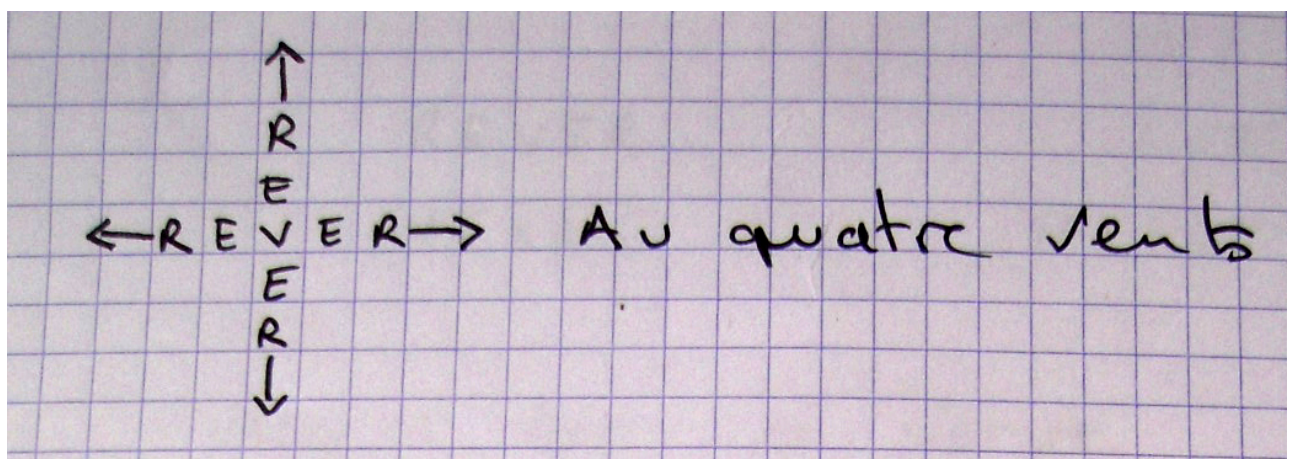

Fig. 6 : RÊVER au quatre vents

\section{« Je t'aime, Ndeysaan » ou l'écriture à voix haute}

Oralittérature ou LittORAL ${ }^{27}$, l'écriture qui se répand sur la page est d'ores et déjà portée - voire canalisée - par le flow du poète, par un rythme régulier et binaire. En témoigne le titre de ce poème doublé d'une micro-alternance en langue peule: "Je t'aime, Ndeysaan ${ }^{28}$ ». Si les vers oscillent de quatre à six syllabes, les rimes sont riches, parfois équivoquées: il s'agit d'une écriture paronomastique (Frontier) au sein de laquelle «femme des cités » se retrouve en filigrane et en écho dans " femme décidée », écriture pour l'œil comme pour l'oreille si l'on observe les anagrammes («voilée »/«violée »). Cette écriture marquée par la paronomase l'est aussi par un formulisme caractéristique de la poésie orale: «Le formulisme en poésie est donc redondance fortement fonctionnalisée, et formellement stylisée ", conclut Zumthor (1987, p. 221). 
Notons que ce manuscrit (fig. 7) constitue la trame de la version enregistrée et diffusée en ligne sur la page Myspace du slameur. Les quelques annotations rendent compte d'inversions entre deux vers - matérialisées par des flèches - visant le respect des rimes alternées. Cependant, ces inversions ne sont pas effectives dans la version oralisée de ce poème-liste, qui reste conforme à l'ordre initialement déterminé. Les croix correspondent aux ajouts de la formule peule "Ndeysaan", tandis que "je t'aime » s'ajoute aux autres vers. Quant au «a » de «famille ", superposé à «femm- ", il nous semble potentiellement révélateur d'un lapsus ou d'un néologisme (femmille) qui affleure ici (par insertion de "femme» à «famille»). Ce poète se distingue donc par une créativité aux multiples facettes : « Mère noire » (Mer noire) pourra-t-on entendre dans la suite d'un slam fleuve qui nous mènera jusqu'aux rivages d'un univers poétique foisonnant...

Fig. 7

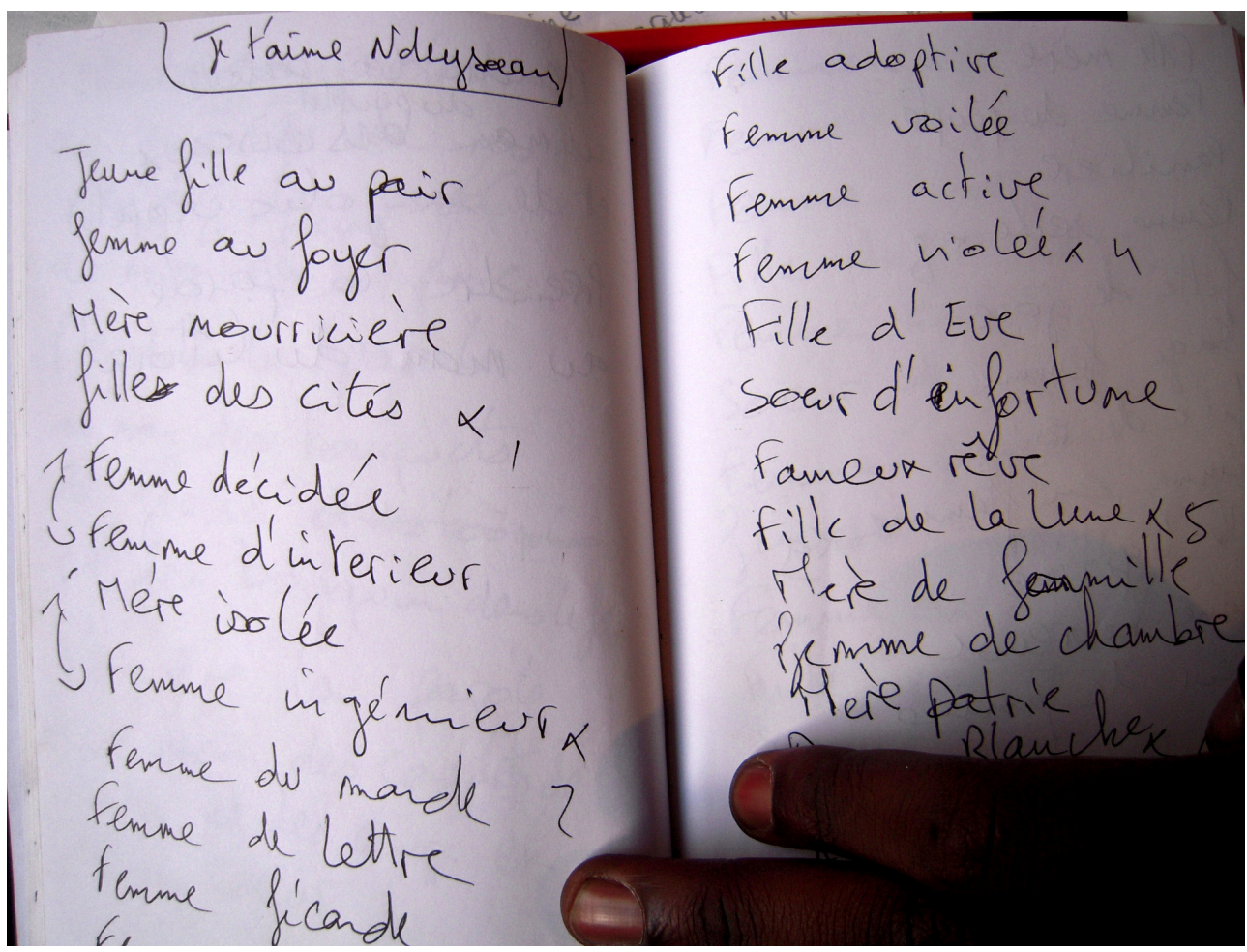

Fig. 7 : Ndeysaan

\section{« Désert de cinq pieds » ou l'écriture à deux voix}

Cet autre échantillon procède d'une écriture à deux mains ou à deux voix (Souleymane Diamanka et John Banzaii), initialement destinée à être oralisée lors d'une émission de radio diffusée sur France Culture. Les pages de manuscrit ont été publiées par Chantal Vieuille pour les éditions Complicités en guise de prologue et d'épilogue au recueil (2007). De fait, une forte complicité unit « le Peul aux yeux bleus et le polonais à la peau noire » qui se plaisent à mêler leurs encres, leurs voix et leurs langues ${ }^{29}$. Il en résulte "deux écritures (qui) peuvent se conjuguer pour n'en faire qu'une", au dire de l'éditrice et sous la plume de nos poètes : «Les jumeaux impossibles/Diseurs de jeux de mots impensables/Réaniment les ratures mortes» (2007, p.42). Notons que le 
palimpseste de «nature morte » fait encore référence à l'art pictural et que c'est un manuscrit peu raturé mais bien coloré qui s'offre à nos yeux: si l'encre noire matérialise la voix de SD, l'encre bleue reflète celle de son « frère de slam ».

Le poème correspondant excède le manuscrit ; il a d'ailleurs fait l'objet d'une réécriture qui vient corroborer notre hypothèse initiale d'une mouvance inhérente aux textes de slam comme à toute poésie "performée »: «Le texte à performance libre [...] n'est jamais deux fois le même: sa surface est comparable à celle d'un lac sous le vent " (Zumthor, 1983, p. 148) ${ }^{30}$. «J'ai nommé la mouvance, désignant ainsi l'instabilité radicale du poème ", ajoute le médiéviste (1983, p. 252, nous soulignons).

21 À travers une telle écriture se conjuguent deux styles, s'agissant d'une écriture-jeu, d'une expérience duelle conçue comme "une partie d'échecs» selon Souleymane Diamanka $^{31}$. En témoigne ce vers biffé : «la partie s'est tue». Deux types de ratures coexistent ici : la biffure et la "tache" ou "pâté d'encre» (Grésillon, 2006), qui demeure minoritaire. Si l'on considère la formule "Dansons sous l'empreinte ", notons que la biffure attire l'œil et laisse apparaitre ces mots qui « dansent » précisément sur la page et sous l'empreinte du stylo. La formule ${ }^{32}$ a d'ailleurs été partiellement reprise comme titre d'un texte-chanson interprété en duo avec Bertrand Cantat : «Danser sous la tempête ${ }^{33}$ ». Cette écriture évolutive et palimpsestuelle, faite de strates successives, ballottée "au gré des vents musicaux", tend à devenir collective à la faveur des moyens modernes de communication et de partage, d'une dynamique que nous qualifions de colludique.

\section{Des mots ouverts et offerts en partage sur la toile : vers une écriture colludique}

\section{Des manuscrits révélés et diffusés sur la toile}

Certains manuscrits publiés sur la toile (voir supra l'exemple de Nevchehirlian) traduisent l'enjeu d'associer le récepteur, de l'enrôler dans le processus même de la genèse comme dans un jeu auquel on voudrait l'initier. Par là même, il s'agit de le faire pénétrer dans "la petite fabrique de slam», dans une écriture désacralisée et démocratisée. De fait, tout slameur se définit aussi en tant qu'animateur de scènes slam et/ou d'ateliers d'écriture : le slam apparaît ainsi comme l'art et la manière d'une écriture partagée. S'il ne diffuse pas ses manuscrits en dehors du recueil cité, Souleymane Diamanka aime à mettre en scène l'écriture au sein de ses clips. En témoigne celui du poème "Papillon en papier ${ }^{34}$ " où l'on voit d'abord le poète en posture d'écrivant, puis les pages de son bloc-notes apparaissant en arrière-plan tandis que ledit « papillon » s'envole jusqu'à ses destinataires :

Même s'il est né de ma plume, si tu l'as aimé et qu'il t'a plu, ce n'est plus mon poème mais un papillon en papier (2007).

\section{Du manuscrit au webuscrit ${ }^{35}$ : des jeux d'écriture partagés sur la toile}

Souleymane Diamanka s'adonne régulièrement à des jeux d'écriture sur la toile, et ce faisant, le «meilleur ami des mots » stimule la créativité de ses amis facebookiens. À 
titre d'exemple, voici deux reproductions de blocs-notes virtuels ${ }^{36}$ consistant en « exercices de style » proposés par le slameur sur sa page (fig. 8).

Fig. 8

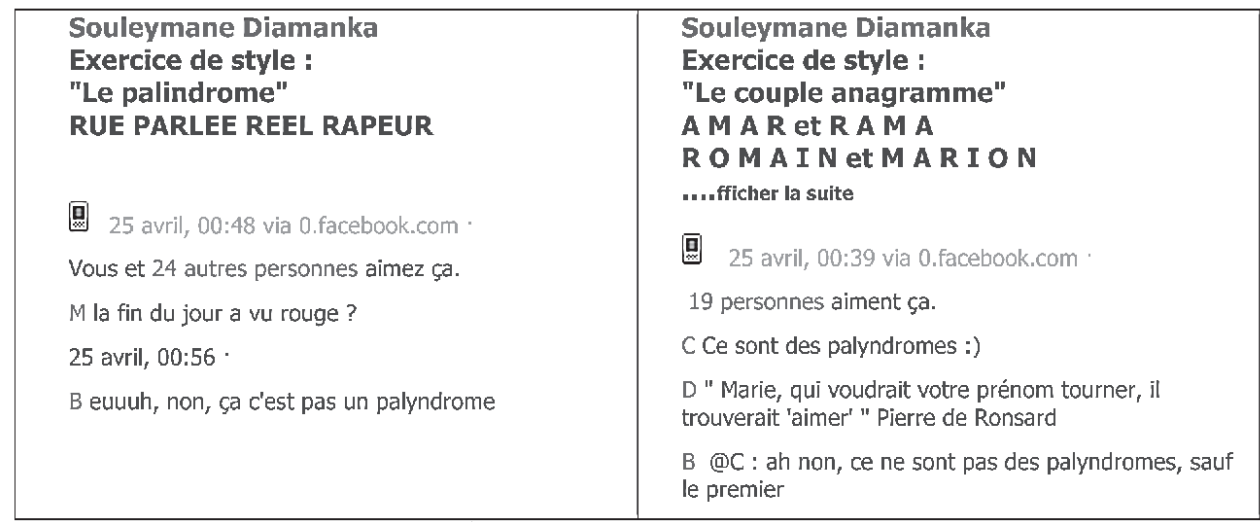

Fig. 8 : Jeux d'écriture proposés sur Facebook (relevés le 25 avril 2011)

Le nombre de commentaires ajoutés par les internautes (dix-huit pour le premier, trente pour le second) qui se prennent au jeu des anagrammes et palindromes est remarquable. C'est à ce que nous traduisons en termes de fonction colludique s'agissant de «jouer ensemble avec les mots »- que le slameur initie ses amis virtuels, en leur montrant les rouages de son écriture et en leur ouvrant ainsi la porte (alors même que le verbe to slam peut signifier " claquer la porte ») de son atelier de poète. Il n'a de cesse d'offrir ses mots en partage, comme s'il cherchait à tester ses vers, aussitôt formulés, allant jusqu'à inciter ses lecteurs-auditeurs à les «mettre en bouche " pour goûter pleinement à la saveur de ses holorimes (fig. 9).

Fig. 9

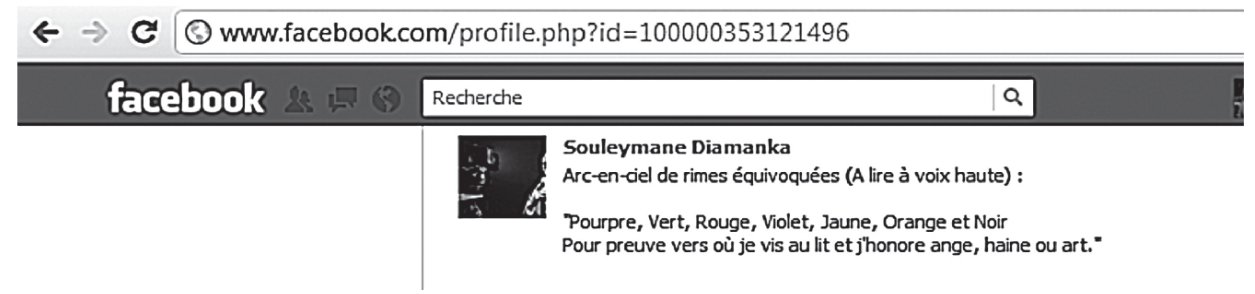

Fig. 9 : L'écriture offerte

Dès lors, le slam serait-il l'art d'offrir les mots en partage et d'impliquer l'auditor in poema?

Dans cette forme de poésie moderne, les « ratures mortes » sont destinées à s'évader du livre, à prendre corps, « encre vivante ${ }^{37}$ » réanimée sur scène par la voix des slameurs... À la suite de Queneau et du verbe "littératurer ${ }^{38}$ ", pourquoi ne pas proposer celui d'«oralittératurer» (mot-monstre à trois têtes!) appliqué au slam? Ce dernier témoigne d'une littérature orale actuelle et en devenir, toujours en marche, de textes à mi-chemin entre l'écriture de leur genèse et l'oralité de leur dessein, poèmes vivants où l'on s'autorise enfin la rature, le retour sur un texte nouveau-né : déjà-là mais toujours mouvant et d'autant plus é-mouvant qu'il s'agit d'un poein en acte, mis en mots et 
marchant "mots dans la main ${ }^{39}$ ", mais aussi mis en bouche, en voix, en corps et en scène. Cet art parfois qualifié de " poésie urbaine » s'avère délibérément moderne dans ses modes de diffusion et de partage au-delà des caractéristiques médiopoétiques (Bobillot, 2012) premières qui renvoient à des traditions plus ou moins ancestrales. Aux cassettes enregistrées en langue peule par son père ${ }^{40}$, le poète répond par une abondance de notes et de mots-perles qu'il parle et offre à l'envi sur ses blocs, carnets bien réels et autres pages virtuelles destinées au partage. Il nous ouvre alors, à travers ces traces d'une écriture palimpseste $e^{41}$, une fenêtre sur un processus de création dont le slam révèle et emblématise la dynamique contemporaine "qui aboutit à faire du processus créatif le concept même de l'œuvre, son centre de gravité mobile, [...] conçue en termes d'intentionnalité, d'inachèvement et de virtualité »(de Biasi, 2000, p. 124). Fenêtre qui ouvre un véritable horizon en matière de didactique de l'écriture.

\section{Références bibliographiques}

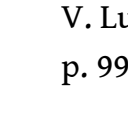
p. 99-164.

вовіLloт J.-P., 2012, « Naissance d'une notion : la médiopoétique », dans Poésie \& Médias,

\section{Actes du colloque de Paris IV-Sorbonne, Paris, éd. du Nouveau Monde, p. 155-173.}

CONFIANT R. et CHAMOISEAU P., 1999, Lettres créoles, Paris, Gallimard.

DEBRAY-GENETTE R., 1988, Métamorphoses du récit, Paris, Éditions du Seuil.

FRONTIER A., 1992, La Poésie, Paris, Belin.

GENETTE G., 1987, Seuils, Paris, Éditions du Seuil.

GRÉSILLON A., 1994, Éléments de critique génétique. Lire les manuscrits modernes, Paris, PUF.

- 2006, «Raturer, rater, rayer, éradiquer, radier, irradier», article en ligne: $<$ www.item.ens.fr/index.php ?id =13973>.

GRÉSILLON A., MERVANT-ROUX M.-M. et BUDOR D. (dir.), 2010, Genèses théâtrales, Paris, CNRS Éditions.

HAGÈGE C., 1987, L'Homme de parole. Contribution linguistique aux sciences-humaines, Paris, Fayard.

LÉGER N. et GRÉSILLON A. (dir.), 2005, Genesis, n²6, « Théâtre ».

VORGER C., 2011, « Le slam est-il néologène ? », Neologica, n 5, p. 73-90.

ZUMTHOR P., 1983, Introduction à la poésie orale, Paris, Éditions du Seuil.

- 1987, La Lettre et la voix, Paris, Éditions du Seuil.
D. et VORGER C., 2011, "Du rap au slam, le flow ne se tarit pas », Synergie Espagne,

. 


\title{
Slamographie
}

\section{Sitographie} productions.

BANZAÏ J. et DIAMANKA S., 2007, J'écris en français dans une langue étrangère [recueil], Paris, Les éditions Complicités.

DIAMANKA S., 2007, L'Hiver peul, Universal music [album CD].

GRAND CORPS MALADE, 2008, Enfant de la ville [CD], Éditions Raoul Breton/Anouche

NEVCHEHIRLIAN, 2005, Vibrion [CD], Autoproduit.

ROUDA, 2007, Musique des Lettres [CD], Harmonia Mundi.

\author{
<http://souleymanediamanka.fr/> \\ $<$ http://nevchehirlian.over-blog.fr/>
}

\section{NOTES}

1. J. Banzaï et S. Diamanka, 2007, « L'homme en ?uestion », p. 64.

2. Nous nous référons ici au titre, néologique, du dernier album du slameur allemand Bas Böttcher (Neonomad, 2009) qui se présente comme poète nomade, à l'instar des aèdes, mais jouissant cependant des nouveaux modes de communication et d'écriture (entretien du 14 octobre 2010).

3. Contrairement à la tradition américaine, l'improvisation est rare dans le slam français.

4. Voir à ce sujet Genesis, $n^{\circ} 26$, "Théâtre ", 2005, et aussi: A. Grésillon et al. (dir.), Genèses théâtrales, Paris, CNRS Éditions, 2010.

5. C'est en termes de «changement d'états " que le slameur Bas Böttcher décrit le slam : le bloc de glace représente la fixité du texte écrit; l'état liquide traduit le flow oral, l'état gazeux la performance.

6. Les entretiens que nous avons réalisés convergent vers l'idée que seule la publication sous la forme d'un $\mathrm{CD}$ commercialisé tend à figer le texte, alors que sa "publication » sur une scène slam autorise a contrario des réécritures selon le feed-back de l'auditoire, une rythmique qui se cherche et se trouve dans la mise en voix.

7. Les manuscrits reproduits sont issus de la collection privée du slameur, avec son aimable autorisation.

8. Propos recueillis le 24 septembre 2010.

9. Marc Smith, ancien ouvrier du bâtiment et poète à ses heures, a créé le concept dans le Chicago des années quatre-vingt.

10. L'une des règles inhérentes à ce dispositif implique que le poème soit " performé » dans une durée variant de trois à cinq minutes : la concision est donc de mise, pour permettre le partage de la scène. 
11. Un slameur grenoblois (le bien nommé « Mots Paumés ») nous a confié écrire " par post-it », soit par bribes, et le plus souvent en mouvement, dans des lieux divers imposés par le statut de " poète nomade".

12. Entretien du 22 novembre 2009. Le livret accompagnant la première édition de l'album de son groupe Vibrion (2005) présentait une véritable architecture visuelle, mise en espace qui n'a pas été maintenue dans la seconde édition. Les pages de carnet visualisées pendant l'entretien matérialisent une certaine dispersion (une phrase = une page). On peut émettre l'hypothèse que l'écriture - en l'occurrence, une écriture-fleuve emblématique du flow du poète - permet d'anticiper sur les effets de mise en voix, la densité étant corrélée au rythme ou à l'intensité. Voir par exemple «La mer», dont le manuscrit est disponible sur son blog: http:// nevchehirlian.over-blog.fr/.

13. Nous faisons ici référence au titre de Marinetti et aux manifestes futuristes proclamant l'avènement d'une poésie où les mots se suivent comme autant de flash visuels, se dispersent ou s'enchevêtrent sur la surface de la page, libérés de toutes contraintes syntaxiques et même de toute successivité.

14. Selon la formule du slameur parisien Rouda (collectif $129 \mathrm{H})$.

15. D'après son fondateur, le slam se veut inclusif («inclusive »), ce qui n'est pas sans effet sur ses caractéristiques lexico-poético-stylistiques et discursives (voir notre article dans Neologica, 2011). 16. Il est l'auteur d'un album (2007) et d'un recueil écrit avec John Banzaï (voir infra et citation mise en exergue).

17. Je n'ai jamais appris à écrire ou Les incipit, Genève, Skira, 1969, p. 12.

18. " GRIFOUILLIS dans le fouillis gris... ", dans Soleil de nuit, Paris, Gallimard, 1980.

19. Nous reprenons ici le titre d'un documentaire (Jérôme Thomas, 2009) consacré à ces formes d'écriture modernes que représentent le rap, le slam, le graffiti : <www.youtube.com/watch? $\mathrm{v}=2 \mathrm{xbRy}_{-}$JUTc>.

20. D'où le slam « Soleil Jaune », dont le titre fait écho à Souley(mane) et John (Banzaï).

21. Le jeu télévisé «Slam » (créé à l'été 2009) consiste en une grille de mots croisés à remplir à l'antenne: le candidat qui s'engage à "slamer» doit remplir toute la grille sans qu'aucune définition ne lui soit donnée. Quant au jeu « Boogle slam », il s'agit d'un jeu de cartes-lettres que les participants doivent mettre à profit pour créer le plus possible de mots à partir de celles d'un mot initial (« mare » devenant « mari, tari, tard», etc.).

22. Nous utilisons ici ce terme en l'opposant au "manuscrit» pour désigner une écriture ébullition: "C'est le brouillon, avec ce que l'étymologie du terme évoque à la fois de boue et d'ébullition " (Grésillon, 1994).

23. D'après The Radiant Child, documentaire de Tamara Davis, 2010.

24. «Les fresques murales peuvent être comparées à des pages d'écriture » (Bazin, 1995, p. 181).

25. « Paradoxalement, la rature est à la fois perte et gain [...] son geste négatif se transforme pour le généticien en un trésor de possibles, sa fonction d'effacement donne accès à ce qui aurait pu devenir texte » (Grésillon, 1994, p. 67).

26. Nous proposons ce néologisme en référence au verbe énoncé par Queneau «littératurer» (voir infra).

27. Tel sera le titre du prochain album de Souleymane Diamanka (entretien cité), qui resémantise ainsi, à travers cette néographie visant à faire ressortir le morphème " oral », le lexème attesté « Littoral».

28. Poème conçu pour la journée des femmes et dont l'enregistrement figure sur la page Myspace du slameur (<www.myspace.com/souleymanediamanka>), mais n'a fait l'objet d'aucune publication écrite à ce jour.

29. Le concept du «Meilleur ami des mots " consiste précisément en un duo où les slameurs tendent à dépasser les frontières des langues : «Je m'adresse à lui en peul, il me répond en polonais, puis on inverse, je parle polonais et il parle peul. Après, on parle en même temps sauf 
que la phrase commence en peul et se termine en polonais. Les deux trucs se fondent, les deux langues se fondent l'une dans l'autre... » (entretien cité).

30. De fait, ce poème a été publié sous deux formes et deux titres : "Désert de cinq pieds » (version ici présentée et publiée dans le cadre du recueil J'écris en français dans une langue étrangère, 2007) a été réécrit pour l'album L'Hiver peul (2007) sous le titre « Soleil Jaune».

31. Entretien cité : «On met une feuille sur la table,j'écris une phrase, tu en écris une autre... »

32. Nous faisons ici référence à ce que Zumthor qualifie de « formulisme » (voir supra).

33. Clip accessible sur <http://souleymanediamanka.fr/>. Notons la parenté sonore entre ces deux mots : empreinte/tempête.

34. Clip accessible sur <http://souleymanediamanka.fr/>.

35. Nous n'utilisons pas ce terme dans son sens le plus courant de «manuscrit provisoirement publié sur la toile, dans l'attente d'un éditeur", mais au sens plus large de "manuscrit ou brouillon empruntant le canal d'Internet (réseaux sociaux de type Facebook) et exploitant les potentialités qui s'y rattachent ».

36. Le fait que le slameur manipule ici son téléphone portable et non le clavier d'ordinateur nous conforte dans cette idée qu'il s'agit là, en quelque sorte, d'une forme moderne et interactive de carnet ou « bloc-notes".

37. Titre d'un autre poème du recueil cité (John Banzaï et Souleymane Diamanka, 2007).

38. Queneau, Le Chiendent, Paris, Gallimard, 1956, p. 295.

39. Expression utilisée par le slameur Rouda, qui renvoie aussi à la constellation médiologique (Bobillot, 2012) propre au slam et qui le distingue de la poésie médiévale ou de l'improvisation pure : la triade main-bouche-oreille.

40. Voir à ce sujet l'émission "Les objets ", diffusée sur France Culture, où le poète a rappelé l'importance de ces enregistrements paternels (diffusée le 25 juillet 2011).

41. Nous avons d'ailleurs montré que le "palimpseste», en termes galissonniens, soit le détournement d'une unité phraséologique longue et plus ou moins complexe, était l'un des traits majeurs de cette écriture (2011).

\section{RÉSUMÉS}

Le slam se définit comme une forme moderne d'oralittérature. Ancré dans des traditions ancestrales liées à l'oralité, destiné à être déclamé en public, il est d'abord « encré » sur le papier, les mots courant sur la page avant d'être offerts en partage. Or les slameurs sont à ce point dans l'écriture partagée que certains d'entre eux nous ont donné à voir, lors d'entretiens menés dans le cadre de notre recherche, leurs manuscrits, carnets et autres blocs-notes, à commencer par le poète Souleymane Diamanka dont les écrits préparatoires se trouvent au cœur du présent article. Autant de « grifouillis » (Aragon) qui sont le lieu de jeux graphiques, jeux de lettres et exercices de styles témoignant d'une écriture en gestation. Cette écriture que l'on pourrait qualifier de «vocale », tant la finalité scénique est prégnante, est aussi une écriture palimpseste faite de réécritures successives. Encre vivante, mouvement « colludique » dont l'un des prolongements et mode de publication est la diffusion de ces jeux d'écriture sur la toile. Ainsi le slameur nous a-t-il laissé entrevoir son atelier, lieu d'un poiein en acte et d'un acte qui tend à intégrer le destinataire, l'auditor in poema. 
Slam defines itself as a modern form of Oraliterature. Rooted in ancestral traditions linked to Orality, meant to be declaimed in public, it is first of all "inked" on the paper, the words flowing on the page before being shared. In fact slammers are so involved in shared writing that during the interviews for our research some of them showed us their manuscripts, notebooks and writing pads, to begin with, the poet Souleymane Diamanka whose preparatory writings are the crux of the present article. So many "grifouillis" (Aragon's playful deformation of gribouillis, meaning scribbles) with graphic games, plays on letters and stylistic exercises showing a writing in gestation. This writing, that might be called "vocal" because the performance finality is so obvious, is also a palimpsest writing made of successive writings. Live ink, "co-ludic" movement of which one of the extensions and means of publication is the diffusion of these writing games on the Net. In this way the slammer gave us a glimpse of his studio, the place of a poiein in actuality and an act that tends to integrate its addressee, the auditor in poema.

El Slam se define como una forma moderna de oraliteratura. Arraigado en tradiciones ancestrales vinculadas a la oralidad, destinado a ser declamado en público, es inicialmente "entintado" en papel ; las palabras desfilan en las páginas antes de ser compartidas. Ahora bien, los slamadores practican hasta tal punto la escritura compartida que algunos de ellos nos han dejado ver, durante las entrevistas realizadas en el marco de nuestra investigación, sus manuscritos, cuadernos y otras libretas de notas; en particular, el poeta Souleymane Diamanka cuyos escritos preparatorios constituyen el núcleo de este artículo. Todos estos "grifoullis" (Aragon) constituyen un campo de juegos gráficos, juegos con letras y ejercicios de estilo qui dan testimonio de una escritura en gestación. Esta escritura, que podríamos calificar de "vocal" dada la importancia de su finalidad escénica, constituye también una escritura palimpsesto hecha de reescrituras sucesivas. Tinta viva, movimiento cuya prolongación y modo de publicación es la difusión de esos juegos de escritura en la red. Así, el eslamador nos ha dejado entrever su taller, lugar de un poiein en acto y de un acto que tiende a integrar al destinatario, al auditor in poema.

Die Slam-Poesie wird hier als eine Form moderner Sprechdichtung definiert. Slam-Texte sind in den Traditionen mündlicher Kulturen verwurzelt und werden für mündliche Performance-Akte verfasst. Sie werden auf Papier konzipiert, bevor sie auf der Bühne mit dem Publikum geteilt werden. Slam-Poeten visieren dermaßen die (mit-)geteilte Literatur, dass uns einige von ihnen anlässlich von Interviews Einblick in ihre handschriftlichen Aufzeichnungen und Notizblöcke gaben. So z.B. Souleymane Diamanka, dessen Vorarbeiten hier im Zentrum stehen. Es sind oft die von Aragon als "griffouillis“ bezeichneten visuellen und akustischen Wortspiele, die Zeugnis ablegen von schöpferischer Schreibarbeit. Slam-Poesie könnte als „Stimme“ verstanden werden, die erst auf der Bühne ihre eigentliche Bestimmung findet. Sie unterliegt ständigen Neubearbeitungen und Veränderungen - lebendig gewordene Tinte, die bis hin zur Performance und zur „Veröffentlichung“ im Internet reicht. So geben uns die Slam-Poeten Einblick in ihre Werkstatt, jenen Ort des poiein, das den Rezipienten als „auditor in poema“ in den Schaffensprozess integriert.

O slam define-se como uma forma moderna de oratura. Ancorado em tradições ancestrais da oralidade e destinado a ser declamado em público, é primeiro escrito sobre papel, as palavras correndo sobre a página antes de serem partilhadas. Os poetas de slam acreditam a tal ponto na escrita partilhada que alguns deles nos revelaram os seus manuscritos, cadernos e blocos, a começar pelo poeta Souleymane Diamanka, cujos escritos preparatórios fundamentam o presente artigo. Aproximam-se dos grifouillis de Aragon, espaço de jogos gráficos, jogos de letras e exercícios estilísticos que testemunham a gestação da escrita. Esta escrita, que poderia ser qualificada de "vocal", tamanha é a sua vocação cénica, é também uma escrita em palimpsesto, que sucessivas reescritas constroem. Tinta viva, movimento "co-lúdico" que tem por prolongamento e modo de publicação a divulgação de tais jogos de escrita sobre a tela. Assim, o 
poeta de slam deixa-nos entrever o seu atelier, espaço de um poiein em acto e de um acto que tende a integrar o destinatário, o auditor in poema.

Lo slam si definisce come una forma moderna di "letteratura orale". Ancorato nelle tradizioni ancestrali legate all'oralità, destinato a essere declamato in pubblico, è "inchiostrato" sulla carta e le parole corrono sulla pagina prima di essere condivise. Gli slamers sono a tal punto legati alla scrittura condivisa che alcuni, nel corso delle interviste condotte nell'ambito della ricerca, ci hanno mostrato i loro manoscritti, quaderni e bloc-notes, a cominciare dal poeta Souleymane Diamanka, i cui scritti preparatori sono il cuore di questo articolo. Così come i grifouillis (Aragon), che sono il luogo di giochi grafici, di lettere e esercizi di stile, testimoniano una scrittura in gestazione. Questa scrittura, che si potrebbe qualificare come "vocale", tanto la finalità scenica è pregnante, è anche una scrittura a palinsesto, fatta di riscritture successive. Inchiostro vivente, movimento "co(l)ludico", del quale uno dei prolungamenti e modi di pubblicazione è la diffusione di questi giochi di scrittura sulla tela. Così lo slamer ci lascia intravedere il suo atelier, luogo di un poiein in atto, e di un atto che tende a integrare il destinatario, l'auditor in poema.

\section{INDEX}

Mots-clés : Slam, manuscrits, critique génétique, carnet, bloc-notes, oral, Diamanka Souleymane, poésie, rature

\section{AUTEUR}

\section{CAMILLE VORGER}

CAMILLE VORGER est docteure ès Linguistique et didactique, auteure d'une thèse intitulée «Poétique du slam : de la scène à l'école » et sous-titrée « Néologie, néostyles et créativité lexicale » (2011) : première thèse en France consacrée à cette forme de poésie contemporaine, envisagée selon une perspective poético-lexicale et didactique, s'agissant d'analyser la créativité à l'œuvre dans et autour du slam et la façon dont elle stylisée dans les textes. Elle est Maître d'Enseignement et de Recherche à l'École de français langue étrangère de la faculté des lettres de l'université de Lausanne.

camille.vorger[arobase]gmail.com 